\title{
WAVELETS ON THE 2-SPHERE AND RELATED MANIFOLDS
}

\author{
J.-P. ANTOINE and P. VANDERGHEYNST* \\ Institut de Physique Théorique, Université Catholique de Louvain, \\ B-1348 Louvain-la-Neuve, Belgium \\ (e-mail: Antoine@fyma.ucl.ac.be) \\ (Received March 24, 1998 - Revised June 22, 1998)
}

\begin{abstract}
We present a group-theoretical derivation of the continuous wavelet transform (CWT) on the 2-sphere $S^{2}$, based on the construction of coherent states associated to square integrable group representations. The parameter space $X$ is the product of $S O(3) \times \mathbb{R}_{*}^{+}$, embedded into the Lorentz group $S O_{o}(3,1)$ via the Iwasawa decomposition, and $X \simeq S O_{o}(3,1) / \mathrm{C}$. The space $L^{2}\left(S^{2}, d \mu\right)$ carries a unitary irreducible representation of $S O_{o}(3,1)$, which is square integrable over $X$, and thus yields the wavelets on $S^{2}$ and the associated CWT.
\end{abstract}

\section{The problem}

In most cases of physical interest, experimental data are given on the line (signal processing), on the plane (image analysis), or occasionally in $\mathbb{R}^{3}$ (e.g. in fluid dynamics). However, there are situations where data are given on a sphere, for instance, geophysical data, statistical problems, computer vision or medical imaging (see [1] for precise references). The standard methods are based on Fourier analysis, but analyzing data with the continuous wavelet transform (CWT) is by now a well-established procedure (see [2] for a survey of applications in physics). So the question arises, how does one extend the CWT to the sphere or another manifold?

Let us first make that statement precise. We may speak of a genuine spherical CWT if (i) the signals and the wavelets live on the sphere; (ii) the transform involves (local) dilations of some kind; and (iii) for small scales, the spherical CWT reduces to the usual CWT on the (tangent) plane (Euclidean limit).

The problem has attracted a lot of interest in the last couple of ycars and many proposals have been made, but, in our opinion, none of them is fully satisfactory. For instance:

- One may extend to $S^{2}$ the discrete wavelet scheme based on a multiresolution analysis, but this approach leads often to numerical difficulties around the poles [3-6]. A different technique is to use second generation wavelets [7].

- One may exploit the geometry of the sphere, as encoded in the system of spherical harmonics [8-11], but the resulting analyzing functions are poorly localized and do not really resemble wavelets.

*Present address: Signal Processing Laboratory, Swiss Federal Institute of Technology (EPFL), CH-1015 Lausanne, Switzerland; e-mail: Pierre.Vandergheynst@epfl.ch 
- In order to avoid the problem of defining proper dilations on the sphere, one defines a WT on the tangent bundle of the sphere [12] or instead a Gabor transform on the sphere itself [13].

- The most satisfactory approach is that of Holschneider [14], who produces a CWT on $S^{2}$ that satisfies the three criteria above. However, the rôle of dilations is played by an abstract parameter that satisfies a number of ad hoc assumptions. The correct Euclidean limit is obtained, but it is essentially put by hand.

As can be seen from this brief description, none of the proposed solutions fully qualifies for a genuine CWT on $S^{2}$. We will present here a new approach to the CWT on the 2-sphere, entirely derived from group theory, following the formalism of general coherent states developed in [15]. In particular, the Euclidean limit is obtained by a group contraction. A detailed treatment may be found in [16] and $[17]$.

\section{General set-up: the CWT on a manifold}

We begin by a brief sketch of the method of construction of coherent states (CS) associated to a group representation. Further details may be found, for instance, in the review paper [15] and the references quoted there.

Let $Y$ be a manifold, such as space $\mathbb{R}^{n}$, the 2 -sphere $S^{2}$, space-time $\mathbb{R} \times \mathbb{R}$ or $\mathbb{R}^{2} \times \mathbb{R}$, etc. In order to construct coherent states on $Y$, assume there is a (locally compact) group $G$ of transformations acting transitively on $Y$. Then $G$ has a natural unitary representation in the space $L^{2}(Y, d \mu)$ of finite energy signals living on $Y$, namely the left quasi-regular representation (for simplicity, we assume that $\mu$ is $G$-invariant):

$$
[U(g) f](y)=f\left(g^{-1} y\right)
$$

Then a system of CS on $Y$ associated to $G$ may be defined if $U$ is square integrable, that is, $U$ is irreducible and there exists a nonzero vector $\eta \in L^{2}(Y, d \mu)$, called admissible, such that the matrix element $\langle U(g) \eta \mid \eta\rangle$ is square integrable as a function on $G$, with respect to the (left or right) Haar measure on $G$. When this is the case, the corresponding CS, indexed by $G$, are the vectors $\eta_{g}=U(g) \eta, g \in G$. Quite often, however, the UIR $U$ is not square integrable in the strict sense, but it becomes so when restricted to a homogeneous space $X=G / H$, for some closed subgroup $H$. More precisely, given a Borel section $\sigma: X \rightarrow G$, the nonzero vector $\eta \in L^{2}(Y, d \mu)$ is said to be admissible $\bmod (H, \sigma)$, and the representation $U$ square integrable $\bmod (H, \sigma)$, if the following condition holds (we assume that $\nu$ is a $G$-invariant measure on $X$ ):

$$
0<\int_{X} d \nu(x)|\langle U(\sigma(x)) \eta \mid \phi\rangle|^{2}=\left\langle\phi \mid A_{\sigma} \phi\right\rangle<\infty, \quad \forall \phi \in \mathcal{H},
$$

where $\Lambda_{\sigma}$ is a positive, bounded, invertible operator [15]. Then CS indexed by $X$ may be defined as

$$
\eta_{\sigma(x)}=U(\sigma(x)) \eta, \quad x \in X
$$


If the operator $A_{\sigma}^{-1}$ is also bounded, the family $\mathcal{S}_{\sigma}=\left\{\eta_{\sigma(x)}, x \in X\right\}$ is called a frame, and a tight frame if $A_{\sigma}=\lambda I$, for some $\lambda>0$. This terminology is familiar in the discrete case, for instance, in wavelet or Gabor analysis [18, 19]. From now on, we shall assume for simplicity that the admissible vector $\eta$ generates a frame $\mathcal{S}_{\sigma}$.

Under these assumptions, the CS defined in (2.3) form a total set in $\mathcal{H}$, i.e. $\left(\mathcal{S}_{\sigma}\right)^{\perp}=\{0\}$, with the following properties:

(1) The range, $\mathcal{H}_{\eta}$, of the linear map $W_{\eta}: \mathcal{H} \rightarrow L^{2}(X, d \nu)$, given by

$$
\left(W_{\eta} \phi\right)(x)=\left\langle\eta_{\sigma(x)} \mid \phi\right\rangle, \quad \phi \in \mathcal{H},
$$

is complete with respect to the scalar product $\langle\Phi \mid \Psi\rangle_{\eta} \equiv\left\langle\Phi \mid W_{\eta} A_{\sigma}^{-1} W_{\eta}^{-1} \Psi\right\rangle$ and $W_{\eta}$ is unitary from $\mathcal{H}$ onto $\mathcal{H}_{\eta}$.

(2) The orthogonal projection from $L^{2}(X, d \nu)$ onto $\mathcal{H}_{\eta}$ is an integral operator $K_{\sigma}$, and $\mathcal{H}_{\eta}$ is a reproducing kernel Hilbert space of functions; the kernel is given explicitly by $K_{\sigma}(x, y)=\left\langle\eta_{\sigma(x)} \mid A_{\sigma}^{-1} \eta_{\sigma(y)}\right\rangle$.

(3) The map $W_{\eta}$ may be inverted on its range by the adjoint operator, $W_{\eta}^{-1}=W_{\eta}^{*}$ on $\mathcal{H}_{\eta}$, to obtain the reconstruction formula:

$$
W_{\eta}^{-1} \Phi=\int_{X} d \nu(x) \Phi(x) A_{\sigma}^{-1} \eta_{\sigma(x)}, \Phi \in \mathcal{H}_{\eta} .
$$

In other words, the vector $\phi=W_{\eta}^{-1} \Phi$ may be expanded in terms of CS $\eta_{\sigma(x)}$.

A particular case of this construction is that considered, independently, by Gilmore [20] and Perelomov [21], namely the situation where the subgroup $H$ is the subgroup $H_{\eta}$ of $G$ that leaves the admissible vector $\eta$ invariant up to a phase, $U(h) \eta=$ $e^{i \omega(h)} \eta, \quad h \in H_{\eta}$, where $\omega(h)$ is a real-valued function on $H_{\eta}$. Then the admissibility condition (2.2) is independent of the choice of the section $\sigma$, and the frame is always tight, with $A_{\sigma}=I$.

Familiar examples of this construction include the ' $a x+b$ ' group acting on $\mathbb{R}$, which yields the usual 1D continuous wavelets; the Weyl-Heisenberg group, also acting on $\mathbb{R}$, that gives the Windowed Fourier Transform, or Gabor transform; the similitude group of $\mathbb{R}^{n}$, consisting of translations, rotations and dilations, which yields the $n$-dimensional wavelets; or coherent states on the Galilei group or the Poincaré group, both inaccessible to the standard Gilmore-Perelomov method [15].

\section{Affine transformations on the sphere $S^{2}$}

We shall apply this method to the sphere $S^{2}$ and consider the space of finite energy signals $\mathcal{H}=L^{2}\left(S^{2}, d \mu\right)$, where $d \mu(\omega)=\sin \theta d \theta d \varphi$ is the usual (rotation invariant) measure on $S^{2}$. The first step for constructing a CWT on $S^{2}$ is to identify the appropriate transformations. These are of two types:

(i) Motions or displacements, given by elements of the rotation group $S O(3)$, which indeed acts transitively on $S^{2}$, and $S^{2} \simeq S O(3) / S O(2)$. 
(ii) Dilations, that may be derived in two steps. First, dilations around the North Pole are obtained by considering usual dilations in the tangent plane and lifting them to $S^{2}$ by inverse stereographic projection from the South Pole; in polar spherical coordinates $\omega=(\theta, \varphi)$, this gives:

$$
D_{a}^{N}(\theta, \varphi)=\left(\theta_{a}, \varphi\right), \quad \text { with } \quad \tan \frac{\theta_{a}}{2}=a \cdot \tan \frac{\theta}{2} .
$$

Then a dilation around any other point $\omega \in S^{2}$ is obtained by moving $\omega$ to the North Pole by a rotation $\gamma \in S O(3)$, performing a dilation $D^{N}$ as before and going back by the inverse rotation: $D^{\omega}=\gamma^{-1} D^{N} \gamma$. Clearly the dilations act also transitively on $S^{2}$.

Next we have to identify a group of affine transformations on $S^{2}$. First we note that motions $\gamma \in S O(3)$ and dilations by $a \in \mathbb{R}_{*}^{+}$do not commute. Also it is impossible to build a semidirect product $S O(3) \rtimes \mathbb{R}_{*}^{+}$, since $S O(3)$ has no outer automorphisms, and therefore the only extension of $S O(3)$ by $\mathbb{R}_{+}^{+}$is their direct product. A way out is to embed the two factors into the Lorentz group $S O_{o}(3,1)$, by the Iwasawa decomposition:

$$
S O_{o}(3,1)=S O(3) \cdot A \cdot N
$$

where $A \sim \mathbb{R}_{*}^{+} \sim S O_{o}(1,1) \sim \mathbb{R}$ is the subgroup of Lorentz boosts in the $z$-direction and $N \sim \mathbb{C}$ is two-dimensional and abelian.

Alternatively, one may consider the twofold covering $S L(2, \mathbb{C})$ of $S O_{o}(3,1)$, which acts on the tangent plane by homographic transformations. The corresponding Iwasawa decomposition rcads $S L(2, \mathbb{C})=S U(2) \cdot A \cdot N$, where $N$ now corresponds to translations of the plane. Therefore the stability subgroup of the North Pole is the minimal parabolic subgroup $P=M A N$, where $M=S O(2)$ or $U(1)$ is the subgroup of rotations around the $z$-axis, depending on the formulation chosen. Thus we get

$$
S^{2} \simeq S O_{o}(3,1) / P \simeq S O(3) / S O(2) \text {. }
$$

This shows that $S O_{o}(3,1)$ acts transitively on $S^{2}$, and so does $S L(2, \mathbb{C})$, via the inverse stereographic projection.

In order to compute explicitly the action of $S L(2, \mathbb{C})$ on $S^{2}$, one may use the Iwasawa decomposition (3.2). For a pure dilation by $a$, the result is precisely the usual dilation lifted on $S^{2}$ by inverse stereographic projection, given in (3.1).

\section{The continuous wavelet transform on $S^{2}$}

\subsection{Principal series representations of the Lorentz group $S O_{o}(3,1)$}

The next step towards constructing affine CS on $S^{2}$ is to find a suitable UIR of the Lorentz group $S O_{o}(3,1)$ in the Hilbert space $L^{2}\left(S^{2}, d \mu\right)$. Natural candidates are the representations of the continuous principal series $[26,27]$, which are given by the operators:

$$
\left[U^{s}(g) f\right](\omega)=\lambda(g, \omega)^{s / 2} \chi(a) f\left(g^{-1} \omega\right), \quad g \in S O_{o}(3,1), \quad s \in \mathbb{C}, \quad f \in L^{2}\left(S^{2}, d \mu\right),
$$


where $g=\gamma a n$ is the Iwasawa decomposition, $\chi$ is a character of $A$, and the multiplier $\lambda(g, \omega)$ is a Radon-Nikodym derivative (or a 1-cocycle), expressing the fact that the measure $d \mu$ is not invariant under the full group $S O_{o}(3,1)$ :

$$
\lambda(g, \omega)=\frac{d \mu\left(g^{-1} \omega\right)}{d \mu(\omega)}, \quad g \in S O_{o}(3,1) .
$$

Among these, we take the subset of class I representations. These are induced by UIRs of the minimal parabolic subgroup $P=M A N$, which are trivial on $M$ (for these representations, the carrier Hilbert space contains a vector which is invariant under the action of the maximal compact subgroup $S O(3))$. For simplicity, we take the trivial character $\chi(a) \equiv 1$.

The corresponding representation $U^{s}$ of $S O_{o}(3,1)$ is cyclic when $s \neq 0,-1,-2, \ldots$ and it is unitary and irreducible if and only if $\operatorname{Re} s=1$ [27]. We choose $s=1$ and write $U \equiv U^{1}$.

Since we are only interested in the action of dilations and motions, we quotient out the subgroup $N$. Then, introducing a suitable section $\sigma: X=S O_{o}(3,1) / N \rightarrow$ $S O_{o}(3,1)$, we concentrate on the reduced expression

$$
[U(\sigma(x)) f](\omega)=\lambda(\sigma(x), \omega)^{1 / 2} f\left(\sigma(x)^{-1} \omega\right) .
$$

We choose the natural (Iwasawa) section $\sigma_{I}(\gamma, a)=\gamma a, \gamma \in S O(3), a \in A$. Using the action (3.1) of dilations, one gets easily

$$
\lambda\left(\sigma_{I}(\gamma, a), \omega\right) \equiv \lambda(a, \theta)=\frac{4 a^{2}}{\left[\left(a^{2}-1\right) \cdot \cos \theta+\left(a^{2}+1\right)\right]^{2}}, \quad \omega=(\theta, \varphi) .
$$

In addition, from the choice of the section, we have $U\left(\sigma_{I}(\gamma, a)\right)=U(\gamma a)=U(\gamma) U(a)$, and therefore the representation (4.3) factorizes as

$$
\left[U\left(\sigma_{I}(\gamma, a)\right) f\right](\omega)=\left(U_{\mathrm{qr}}(\gamma) \circ D^{a} f\right)(\omega),
$$

where $U_{\mathrm{qr}}(\gamma), \gamma \in S O(3)$ is the quasi-regular representation of $S O(3)$ in $L^{2}\left(S^{2}, d \mu\right)$, and $D^{a}, a \in \mathbb{R}_{*}^{+}$is a pure dilation, that is, $\left(D^{a} f\right)(\omega)=\lambda(a, \theta)^{1 / 2} f\left(\omega_{1 / a}\right)$, with $\omega_{a} \equiv\left(\theta_{a}, \varphi\right)$. The quasi-regular representation of $S O(3),\left(U_{\mathrm{qr}}(\gamma) f\right)(\omega)=f\left(\gamma^{-1} \omega\right)$, is infinite-dimensional and decomposes into the direct sum of all the familiar $(2 l+1)$-dimensional representations, $l=0,1,2, \ldots$.

\subsection{Lorentz coherent states as wavelets on the sphere $S^{2}$}

Following the general approach of [15], we will build in this section a system of CS for the Lorentz group, indexed by points of $X-S O_{o}(3,1) / N$. Since $N$ is not the isotropy subgroup of a particular vector in the representation Hilbert space, the resulting CS are not of the Gilmore-Perelomov type [21]. 
First we show that the UIR (4.3) is indeed square integrable on $X$, that is, we check that there exists a nonzero vector $\eta \in L^{2}\left(S^{2}, d \mu\right)$ such that

$$
\int_{X} d \nu(x)|\langle U(\sigma(x)) \eta \mid \phi\rangle|^{2}<\infty, \quad \forall \phi \in L^{2}\left(S^{2}, d \mu\right),
$$

where $d \nu(\gamma, a)=a^{-3} d \mu(\gamma) d a$, and $d \mu(\gamma)$ is the invariant (Haar) measure on $S O(3)$.

The result is given by the following theorem, proven in [16].

THEOREM 4.1. The representation $U$ given in (4.3) is square integrable modulo the subgroup $N$ and the section $\sigma_{I}$, that is, there exists a nonzero vector $\eta \in L^{2}\left(S^{2}, d \mu\right)$ which is admissible $\bmod \left(N, \sigma_{I}\right)$. This means there is a constant $c>0$, independent of $l$, such that

$$
\frac{8 \pi^{2}}{2 l+1} \sum_{|m| \leqslant l} \int_{0}^{\infty} \frac{d a}{a^{3}}\left|\widehat{\eta}_{a}(l, m)\right|^{2}<c, \quad l=0,1,2 \ldots,
$$

where $\hat{\eta}(l, m)=\left\langle Y_{l}^{m} \mid \eta\right\rangle$ stands for the Fourier coefficients of $\eta$ and

$$
\eta_{a}(\omega)=\left[U\left(\sigma_{I}(e, a)\right) \eta\right](\omega) \equiv\left(D^{a} \eta\right)(\omega)=\lambda(a, \theta)^{1 / 2} \eta\left(\omega_{1 / a}\right) .
$$

As usual, it follows from this theorem that there is a dense set of admissible vectors, and any one of them, $\eta$, generates a continuous family $\left\{\eta_{\sigma_{I}(x)}=U(\sigma(x)) \eta, x \in X\right\}$ of CS. But in fact we have more:

PROPOSITION 4.2. For any admissible vector $\eta$ such that $\int_{0}^{2 \pi} d \varphi \eta(\theta, \varphi) \neq 0$ (for instance, if $\eta$ is axisymmetric), the family $\left\{\eta_{\sigma_{I}(x)}, x \in X\right\}$ is a continuous frame, that is, there exist constants $A>0$ and $B<\infty$ such that

$$
A\|\phi\|^{2} \leqslant \int_{X} d \nu(x)\left|\left\langle\eta_{\sigma_{I}(x)} \mid \phi\right\rangle\right|^{2} \leqslant B\|\phi\|^{2}, \quad \forall \phi \in L^{2}\left(S^{2}, d \mu\right) .
$$

Thus, for most admissible vectors $\eta$, we gct a continuous frame, but not nccessarily a tight frame. We conjecture that the resulting frame is never tight, that is, the frame operator $A_{\sigma}$ has spectrum spread over a nontrivial interval.

Theorem 4.1 yields the basic ingredient for writing the CWT on $S^{2}$. Given an admissible vector $\psi \in L^{2}\left(S^{2}, d \mu\right)$, our wavelets on the sphere are the functions $\psi_{\gamma, a}=$ $U\left(\sigma_{I}(\gamma, a)\right) \psi$, and the CWT reads, with $U_{S}(\gamma, a) \equiv U\left(\sigma_{I}(\gamma, a)\right)$ :

$$
\begin{aligned}
S(\gamma, a) & =\left\langle U\left(\sigma_{I}(\gamma, a)\right) \psi \mid s\right\rangle \\
& =\int_{S^{2}} d \mu(\omega) \overline{\left[U_{S}(\gamma, a) \psi\right](\omega)} s(\omega) \\
& =\int_{S^{2}} d \mu(\omega) \overline{\psi_{a}\left(\gamma^{-1} \omega\right)} s(\omega) .
\end{aligned}
$$


An explicit calculation [16] shows that the spherical CWT (4.9) is covariant under motions on $S^{2}$, but not covariant under dilations. For applications, of course, it is the covariance under motions that is essential, since it reduces to translation covariance in the Euclidean limit, as we shall see in Section 5. As for dilations, the negative result reflects the fact that the parameter space $X$ of the spherical CWT is not a group.

The condition (4.6), which was derived in [14] in a different way, is necessary and sufficient for the admissibility of $\eta$, but it is somewhat complicated to use in practice, since it requires the evaluation of nontrivial Fourier coefficients. Instead, there is a simpler, although only necessary, condition.

PropostTION 4.3. A function $\eta \in L^{2}\left(S^{2}, d \mu\right)$ is admissible only if it satisfies the condition

$$
\int_{S^{2}} d \mu(\theta, \varphi) \frac{\eta(\theta, \varphi)}{1+\cos \theta}=0 .
$$

This necessary condition is the exact equivalent of the usual necessary condition for wavelets in the plane, $\int d^{2} x \psi(x)=0$, and it reduces to the latter in the Euclidean limit (see Section 5). The interesting point is that (4.10) is a zero-mean condition, as in the flat case. As such it has the same effect, namely it ensures that the CWT on $S^{2}$ given in (4.9) acts as a local filter. This is crucial for applications and it is one of the main reasons of the efficiency of the CWT, and the same holds here.

Using Proposition 4.3, it is easy to build explicit wavelets on the sphere, namely 'Difference wavelets', similar to the 'Difference-of-Gaussians' (DOG) wavelet commonly used in vision. Given a square integrable function $\phi$, we define

$$
\eta_{\phi}^{(\alpha)}(\theta, \varphi)=\phi(\theta, \varphi)-\frac{1}{\alpha} D^{\alpha} \phi(\theta, \varphi) \quad(\alpha>1) .
$$

Then it is easily checked that $\eta_{\phi}^{(\alpha)}$ satisfies the admissibility condition (4.10), that is, it is a spherical wavelet. A typical difference wavelet corresponds to the choice $\phi(\theta, \varphi)=\exp \left(-\tan ^{2} \frac{\theta}{2}\right)$, which is the inverse stereographic projection of a Gaussian in the tangent plane.

\section{The Euclidean limit}

According to Holschneider [14], a good wavelet transform on the sphere should be asymptotically Euclidean, that is, the spherical WT should match the usual CWT in the plane at small scales or, what amounts to the same, for large values of the radius of curvature. In this section, we will give a precise mathematical meaning to this statement using the technique of group contractions.

\subsection{Contracting the Lorentz group and its homogeneous spaces}

In the first step, we reformulate the theory on a sphere of radius $R$ and let $R \rightarrow \infty$. In this limit, the Lorentz group $S O_{o}(3,1)$ is contracted into a semidirect product:

$$
G_{1}=S O_{o}(3,1) \stackrel{R \rightarrow \infty}{\longrightarrow} G_{2}=\mathbb{R}^{2} \rtimes \operatorname{SIM}(2),
$$


where $\operatorname{SIM}(2)=\mathbb{R}^{2} \rtimes\left(\mathbb{R}_{*}^{+} \times S O(2)\right)$ is the similitude group of $\mathbb{R}^{2}$, that is, the invariance group of the Euclidean CWT.

Let us start at the level of Lie algebras. Given two Lie algebras $\mathfrak{g}_{1}=\left(V,[.,]_{1}\right)$ and $\mathfrak{g}_{2}=\left(V,[,,]_{2}\right)$ on the same vector space $V$, we say that $\mathfrak{g}_{2}$ is a contraction of $\mathfrak{g}_{1}$ if there exists a family of invertible linear mappings $\phi_{R}: V \rightarrow V, R \in[1, \infty)$, such that

$$
\lim _{R \rightarrow \infty} \phi_{R}^{-1}\left[\phi_{R} X, \phi_{R} Y\right]_{1}=[X, Y]_{2}, \quad \forall X, Y \in V .
$$

We apply this to the Lorentz Lie algebra $\mathfrak{g}=\mathfrak{s o}(3,1)$, with Iwasawa decomposition:

$$
\mathfrak{s o}(3,1)=\mathfrak{s o}(3) \oplus \mathfrak{a} \oplus \mathfrak{n} .
$$

The minimal parabolic subalgebra is $\mathfrak{p}=\mathfrak{s o}(2) \oplus \mathfrak{a} \oplus \mathfrak{n}$, which is isomorphic to $\mathfrak{s i m}(2)=$ $\mathfrak{s o}(2) \oplus \mathbb{R} \oplus \mathbb{R}^{2}$, the Lie algebra of $\operatorname{SIM}(2)$. Introducing generators of $\mathfrak{s o}(3,1)$, we have :

$$
\mathfrak{s o}(3)=\operatorname{span}\left\{X_{1}, X_{2}, X_{3}\right\}, \quad \mathfrak{a}=\operatorname{span}\{Q\} \sim \mathbb{R}, \quad \mathfrak{n}=\operatorname{span}\left\{N_{1}, N_{2}\right\} \sim \mathbb{R}^{2} .
$$

Then we introduce the following contraction scheme

$$
\phi_{R}\left(X_{j}\right)=R^{-1} X_{j}, \quad j=1,2, \text { all the others fixed. }
$$

When $R \rightarrow \infty$, this contraction preserves the subalgebra $\mathfrak{p}$ and maps $\mathfrak{s o}(3,1)$ onto

$$
\mathfrak{g}_{2}=\mathbb{R}^{2} \oplus \mathfrak{s o}(2) \oplus \mathbb{R} \oplus \mathbb{R}^{2}=\mathfrak{v}_{c}+\mathfrak{s},
$$

where $\mathfrak{v}_{c}=\operatorname{span}\left\{X_{1}, X_{2}\right\} \sim \mathbb{R}^{2}$ and $\mathfrak{s}=\operatorname{span}\left\{X_{3}, Q, N_{1}, N_{2}\right\} \sim \operatorname{sim}(2) \sim \mathfrak{p}$.

Next the contraction procedure is lifted to the corresponding Lie groups. Let $S$ be the subgroup of $G_{1}=S O_{o}(3,1)$ with the Lie algebra $\mathfrak{s}$, and $V_{c}=\exp \mathfrak{v}_{c} \simeq \mathfrak{v}_{c}$. Then the contraction maps $G_{1}=S O_{o}(3,1)$ onto the group $G_{2}$ corresponding to $\mathfrak{g}_{2}$, namely the semidirect product $G_{2} \equiv V_{c} \rtimes S \equiv \mathbb{R}^{2} \rtimes \operatorname{SIM}(2)$. The contraction preserves the minimal parabolic subgroup $P=M A N \sim \operatorname{SIM}(2)$. Concretely, one considers the family of contraction maps $\Pi_{R}: G_{2} \rightarrow G_{1}$ given by

$$
\Pi_{R}:(v, s) \mapsto\left(\exp _{G_{1}} R^{-1} v\right) \cdot s .
$$

They have the same function as the maps $\phi_{R}$ of (5.1) at the group level, namely:

$$
\lim _{R \rightarrow \infty} \Pi_{R}^{-1}\left(\Pi_{R}(g) \stackrel{1}{\circ} \Pi_{R}\left(g^{\prime}\right)\right)=g \stackrel{2}{\circ} g^{\prime}, \quad \forall g, g^{\prime} \in G_{2},
$$

where $\stackrel{1}{\circ}, 2$ denote the product in $G_{1}, G_{2}$, respectively. Indeed $T_{e} \Pi_{R}=\phi_{R}$,

The third step is to transfer the contraction process to the relevant homogeneous spaces. On one hand, the manifolds $S^{2}=G_{1} / M A N$ and $\mathbb{R}^{2}=G_{2} / M A N$, that carry the respective CWT, are related through contraction. On the other hand, since the abelian subgroup $N$ is preserved under the contraction, the parameter space $X=S O_{o}(3,1) / N$ of the spherical CWT goes into that of the Euclidean CWT, namely $\operatorname{SIM}(2)=G_{2} / N$. 
In order to formulate the contraction directly on the two parameter spaces, we introduce a section $\tilde{\sigma}: \operatorname{SIM}(2) \rightarrow N \rtimes \operatorname{SIM}(2)$ by the relation:

$$
\tilde{\sigma}:(\vec{b},(a, \psi)) \mapsto(\vec{n}(\vec{b}),(\vec{b},(a, \psi)))
$$

Combining this with the canonical projection of the Iwasawa bundle, $\Gamma: K A N \rightarrow X \simeq$ $K A$, we define the restricted contraction map $\tilde{\Pi}_{R}: \operatorname{SIM}(2) \rightarrow X$ by

$$
\operatorname{SIM}(2) \ni g \mapsto \tilde{\Pi}_{R}(g)=\Gamma\left(\Pi_{R}(\tilde{\sigma}(g))\right),
$$

where $\Pi_{R}: G_{2} \rightarrow G_{1}$ is the contraction map (5.6). Altogether we have the following commutative diagram:

$$
\begin{array}{cc}
G_{2}=\mathbb{R}^{2} \rtimes \operatorname{SIM}(2) \stackrel{\Pi_{R}}{\longrightarrow} G_{1}=S O_{o}(3,1) \\
\uparrow \tilde{\sigma} & \downarrow \Gamma \\
G_{2} / N \simeq \operatorname{SIM}(2) & \stackrel{\tilde{\Pi}_{R}}{\longrightarrow} S O_{o}(3,1) / N=X .
\end{array}
$$

Finally we notice that the parameter space of the spherical CWT, namely $X=$ $G_{1} / N \simeq S O(3) \cdot A$, is not a group (and this forced us to use the general formalism of [15]), whereas, after contraction, we get $G_{2} / N \simeq \operatorname{SIM(2).~Thus~the~missing~group~}$ structure is restored by the contraction!

\subsection{The Euclidean limit of the spherical CWT}

We may perform now the Euclidean limit itself, that will be formulated as a contraction at the level of group representations. Whereas contractions of Lie algebras and Lie groups are relatively ancient and well known [28, 29], the extension of the procedure to group representations is rather recent [30]. A rigorous version has been given by Dooley [31], which we follow. The additional difficulty here is that the representation space itself varies during the procedure.

Let $G_{2}$ be a contraction of $G_{1}$, defined by the contraction map $\Pi_{R}: G_{2} \rightarrow G_{1}$ and let $U$ be a representation of $G_{2}$ in a Hilbert space $\mathcal{H}$. Suppose that, for each $R \in[1, \infty)$, we have a representation $\left\{\mathcal{H}_{R}, U_{R}\right\}$ of $G_{1}$, a dense subspace $\mathcal{D}_{R}$ of $\mathcal{H}$ and a linear injective map $I_{R}: \mathcal{H}_{R} \rightarrow \mathcal{D}_{R}$. Then one says that the representation $U$ of $G_{2}$ is a contraction of the family of representations $\left\{U_{R}\right\}$ of $G_{1}$ if there exists a dense subspace $\mathcal{D}$ of $\mathcal{H}$ such that, for all $\phi \in \mathcal{D}$ and $g \in G_{2}$, one has:

- for every $R$ large enough, $\phi \in \mathcal{D}_{R}$ and $U_{R}\left(\Pi_{R}(g)\right) I_{R}^{-1} \phi \in I_{R}^{-1}\left(\mathcal{D}_{R}\right)$,

- $\lim _{R \rightarrow \infty}\left\|I_{R} U_{R}\left(\Pi_{R} g\right) I_{R}^{-1} \phi-U(g) \phi\right\|_{\mathcal{H}}=0$.

Using this definition, we show that the CWT on the sphere converges to the usual 2D CWT on $\mathbb{R}^{2}$ in the geometrical limit of large radius, by proving that the associated series of square integrable representations of $S O_{o}(3,1)$ contracts to the usual wavelet representation of $\operatorname{SIM}(2)$, defined in $[23,24]$.

Let $\mathcal{H}_{R}=L^{2}\left(S_{R}^{2}, d \mu_{R}\right)$ be the Hilbert space of square integrable functions on a sphere of radius $R$ and $\mathcal{H}=L^{2}\left(\mathbb{R}^{2}, d^{2} \vec{x}\right)$. Guided by the geometry of the problem, 
we define the map $I_{R}: \mathcal{H}_{R} \rightarrow \mathcal{H}$ as:

$$
\left(I_{R} f\right)(r, \varphi)=\frac{4 R^{2}}{4 R^{2}+r^{2}} f\left(2 \arctan \frac{r}{2 R}, \varphi\right),
$$

using polar coordinates $(r, \varphi)$ in the plane. Then one checks that $I_{R}$ is unitary.

For each $R$, we choose $\mathcal{D}_{R}=\mathcal{D}=\mathcal{C}_{0}\left(\mathbb{R}^{2}\right)$, the space of continuous functions of compact support. Let $U$ be the usual wavelet representation of $\operatorname{SIM(2)}$ in $\mathcal{H}$ and $U_{R}$ the representation $(4.3)$ of $S O_{o}(3,1)$ realized in $\mathcal{H}_{R}$. Then one has

THEOREM 5.1 (Euclidean limit). The representation $U$ of $\operatorname{SIM(2)~is~a~contraction~of~}$ the family of representations $U_{R}$ of $S O_{o}(3,1)$ as $R \rightarrow \infty$.

The proof amounts to show that the strong limit (5.9) holds for every $g \subset \operatorname{SIM}(2)$, with respect to the contraction map $\tilde{\Pi}_{R}$. This is done in two steps. First, an explicit calculation of the map $\check{\Pi}_{R}$ yields pointwise convergence. Then, using the fact that $I_{R}^{-1}$ is a unitary operator, one computes the strong limit in $\mathcal{H}_{R}$, where it results from an easy estimate and an application of Lebesgue's dominated convergence theorem.

This theorem yields the expected result that local wavelet analysis on the sphere as defined here is equivalent to local wavelet analysis in flat space. Indeed, the whole structure on the sphere $S_{R}^{2}$ goes into the corresponding one in $\mathbb{R}^{2}$ as $R \rightarrow \infty$. Since $U_{R} \rightarrow U$, the corresponding matrix elements converge to one another, and so the square integrability condition (4.6) converges into the corresponding one for the CWT in $\mathbb{R}^{2}$,

$$
\int_{\mathbb{R}^{2}} d^{2} k \frac{|\widehat{\psi}(k)|^{2}}{|k|^{2}}<\infty .
$$

Admissible wavelets on $S^{2}$ converge to admissible wavelets on $\mathbb{R}^{2}$, and the necessary condition (4.10) also goes into the usual one in the plane, namely $\int d^{2} x \psi(x)=0$.

\section{Extension to other manifolds}

First we notice that the whole construction made so far extends almost verbatim to the $(n-1)$-dimensional sphere $S^{n-1}=S O(n) / S O(n-1)$, with help of a similar class I representation of the gencralized Lorentz group $S O_{o}(n, 1)$ [32]. Although the spheres are the manifolds on which a CWT is most desirable for applications, the mathematical analysis made here invites to consider other manifolds with similar geometrical properties.

We take first $n=3$. The sphere $S^{2}=S O(3) / S O(2)$ is a compact Riemannian symmetric space of constant curvature $\kappa=1$. It has a noncompact dual, $H^{2}=S O_{o}(2,1) /$ $S O(2)$, of constant curvature $\kappa=-1$ [25]. $H^{2}$ is a two-sheeted hyperboloid, symmetric around the $x_{3}$-axis. Duality corresponds to the fact that $S O(3)$ and $S O_{o}(2,1)$ are the two real forms of the complex group $S O(3)^{\mathbb{C}} \sim S L(2, \mathbb{C})$.

As for the sphere, we can perform a stereographic projection $\Phi$ from the South Pole onto the equatorial plane $x_{3}=0$. Then $\Phi$ maps the upper sheet $H_{+}^{2}$ onto the 
interior $\mathcal{D}_{+}$of the unit disk, and the lower sheet $H_{-}^{2}$ onto the exterior. The domain $\mathcal{D}_{+}$, called the Lobachewskian disk, is conformally equivalent to $H_{+}^{2}$, and both manifolds have $S O_{o}(2,1)$ as isometry group.

Dilations on $H_{+}^{2}$ may be obtained by lifting dilations in the equatorial plane by inverse stereographic projection. The resulting map has all the required properties for a dilation, but does not come directly from a linear group action. Thus it can only be used for constructing wavelets on $H_{+}^{2}$ if one puts it by hand.

An alternative way of introducing dilations via a group action is based on analytic continuation from the spherical case, that is, using the familiar Weyl trick, which in this case maps $\mathfrak{s o}(3)$ to $\mathfrak{s o}(1,2)$. This yields the pseudo-Iwasawa decomposition $G^{*}=S O_{o}(1,3)=S O_{o}(1,2) \cdot A \cdot N^{*}$, which gives the action of $G^{*}$ on $H_{+}^{2}$. It remains to find a UIR $U$ of $S O_{o}(1,3)$ in $L^{2}\left(H_{+}^{2}, d \mu\right)$ and to show that it is square integrable modulo $N^{*}$ and a suitable section.

In higher dimensions, the situation is exactly the same:

$$
S^{n-1}=S O(n) / S O(n-1) \quad \text { and } \quad H^{n-1}=S O_{o}(n-1,1) / S O(n-1)
$$

are dual Riemannian symmetric spaces, with constant curvature $\kappa= \pm 1$, respectively. Again $S O(n)$ and $S O_{o}(n, 1)$ are two real forms of the complexified group $S O(n)^{\mathrm{C}}$. In addition, there are now additional noncompact real forms $S O_{o}(p, q), p+q=n$, leading to pseudo-Riemannian symmetric spaces $H^{p, q}=S O_{o}(p, q) /(S O(p) \times S O(q))$. These are homogeneous spaces of higher rank, and few explicit results are available.

\section{Conclusion}

The construction presented here fulfills all the requirements stated in the introduction for a continuous wavelet transform on the sphere. It is entirely derived from group theory, following the formalism of general coherent states developed in [15]. In addition, the Euclidean limit is valid, with a precise group-theoretical formulation. Thus the formulae (4.9) yield a genuine CWT on the sphere, which has none of the defects of the other versions mentioned above. Preliminary tests with the spherical DOG wavelet show that it has the expected capability of detecting discontinuities, whether or not they lie at one of the poles of the sphere. The only remaining problem is of a computational nature. Indeed Eq. (4.9) requires a pointwise convolution on the sphere, which is very time-consuming. However, this is not specific to wavelet analysis, it simply reflects the lack of an efficient convolution algorithm on the sphere, and in particular the difficulty of finding an appropriate discretization of the latter. Several methods have been proposed in the literature $[1,10,33]$, but none of them is fully satisfactory. However, it seems reasonable to hope that faster algorithms will be available soon [17].

\section{REFERENCES}

[1] D. M. Healy Jr., D. Rockmore and S. S. B. Moore: FFTs for the 2-sphere - Improvements and variations, Techn. Report PCS-TR96-292, Dartmouth College, Hanover, NH 1996.

[2] J. C. van den Berg (Ed.): Wavelets in Physics, Cambridge University Press, Cambridge 1999. 
[3] S. Dahlke, W. Dahmen, E. Schmitt and I. Weinreich: Numer. Funct. Anal. and Optimiz. 16 (1995), $19-41$.

[4] D. Potts and M. Tasche: Interpolatory wavelets on the sphere, in: Approximation Theory VIII, pp. 335-342;

C. K. Chui and L. L. Schumaker (Eds.), World Scientific, Singapore 1995.

[5] J. Göttelmann: Locally supported wavelets on the sphere, preprint, J. Gutenberg-Universität Mainz (1996).

[6] I. Weinreich: Biorthogonale Wavelets auf der Sphäre, Dissertation, RWTH Aachen 1997.

[7] P. Schröder and W. Sweldens: Spherical wavelets: Efficiently representing functions on the sphere, in: Computer Graphics Proc. (SIGGRAPH95), ACM Siggraph 1995, pp. 161-175; W. Sweldens: Applied Comput. Harm. Anal. 3 (1996), 1186-1200.

[8] F. J. Narcowich and J. D. Ward: Applied Comput. Harm. Anal. 3 (1996), 1324-1336.

[9] D. Potts, G. Steidl and M. Tasche: Kernels of spherical harmonics and spherical frames, in: Advanced Topics in Multivariate Approximation; F. Fontanella, K. Jetter and P-J. Laurent (Eds.), World Scientific, Singapore 1996, pp. 1-154.

[10] W. Freeden and U. Windheuser: Applied Comput. Harm. Anal. 4 (1997), 1-37, and papers quoted therein.

[11] W. Freeden, M. Schreiner and T. Gervens: Constructive Approximation on the Sphere, with Applications to Geomathematics, Clarendon Press, Oxford 1997.

[12] S. Dahlke and P. Maass: J. Fourier Anal. Appl. 2 (1996), 379-396.

[13] B. Torrésani: Phase space decompositions: Local Fourier analysis on spheres, preprint CPT-93/P.2878, Marseille (1993); Signal Proc. 43 (1995), 341-346.

[14] M. Holschneider: J. Math. Phys. 37 (1996), 4156-4165.

[15] S. Twareque Ali, J-P. Antoine, J-P. Gazeau and U. A. Mueller: Reviews Math. Phys. 7 (1995), 1013-1104.

[16] J-P. Antoine and P. Vandergheynst: Applied Comput. Harm. Anal. 6 (1999) (to appear).

[17] P. Vandergheynst: Ondelettes directionnelles et ondelettes sur la sphère, Thèse de Doctorat, Univ. Cath. Louvain, Louvain-la-Neuve (1998).

[18] I. Daubechies, A. Grossmann and Y. Meyer: J. Math. Phys. 27 (1986), 1271-1283.

[19] I. Daubechies: Ten Lectures on Wavelets, SIAM, Philadelphia 1992.

[20] K. Gilmore: Ann. Phys. (NY) 74 (1972), 391-463; Rev. Mex. Fis. 23 (1974), 143-187.

[21] A. M. Perelomov: Commun. Math. Phys. 26 (1972), 222-236; Generalized Coherent States and their Applications, Springer, Berlin 1986.

[22] A. Grossmann, J. Morlet and T. Paul: J. Math. Phys. 26 (1985), 2473-2479; Ann. Inst. Henri Poincaré 45 (1986), 293-309.

[23] R. Murenzi: Ondelettes multidimensionnelles et applications à l'analyse d'images, Thèse de Doctorat, Univ. Cath. Louvain, Louvain-la-Neuve (1990).

[24] J-P. Antoine, P. Carrette, R. Murenzi and B. Piette: Signal Proc. 31 (1993), 241-272.

[25] S. Helgason: Differential Geometry, Lie Groups, and Symmetric Spaces, Academic Press, New York 1978.

[26] A. W. Knapp: Lie Groups Beyond an Introduction, Birkhäuser, Basel 1996.

[27] R. Takahashi: Bull. Soc. Math. France 91 (1963), 289-433.

[28] E. Inönü and E. P. Wigner: Proc. Nat. Acad. Sci. U. S. 39 (1953), 510-524.

[29] J. Saletan: J. Math. Phys. 2 (1961), 1-21.

[30] J. Michelsson and J. Niederle: Commun. Math. Phys. 27 (1972), 167-180.

[31] A. H. Dooley: Contractions of Lie groups and applications to analysis, in: Topics in Modem Harmonic Analysis, Vol.I; Istituto Nazionale di Alta Matematica Francesco Severi, Roma 1983, pp. 483-515; A. H. Dooley and J. W. Rice: Math. Proc. Camb. Phil. Soc. 94 (1983), 509-517; Trans. Amer. Math. Soc. 289 (1985), 185-202.

[32] J.-P. Antoine and P. Vandergheynst: J. Math. Phys. 39 (1998), 3987-4008.

[33] M. R. Mohlcnkamp: A fast transform for spherical harmonics, Ph.D. Thesis, Yale University, New Haven 1997. 